\title{
Every breath you take: The value of the electronic nose (e-nose) technology in the early detection of lung cancer
}

\author{
Gaetano Rocco, MD, FRCSEd
}

\footnotetext{
From the Division of Thoracic Surgery, Thoracic Department, Istituto Nazionale Tumori, IRCCS, Fondazione G. Pascale, Naples, Italy.

Received for publication Sept 28, 2017; revisions received Nov 27, 2017; accepted for publication Dec 9, 2017; available ahead of print March 28, 2018.

Address for reprints: Gaetano Rocco, MD, FRCSEd, Division of Thoracic Surgery, Thoracic Department, Istituto Nazionale Tumori, IRCCS, Fondazione G. Pascale, Via Mariano Semmola 81, 80131, Naples, Italy (E-mail: g.rocco@istitutotumori.na.it).

J Thorac Cardiovasc Surg 2018;155:2622-5

$0022-5223 / \$ 36.00$

Copyright (c) 2018 by The American Association for Thoracic Surgery

https://doi.org/10.1016/j.jtcvs.2017.12.155
}

Feature Editor's Note-In this Feature Expert Opinion article, Dr Rocco presents a thoughtfully constructed review detailing the background and significance of an emerging technology in thoracic oncology. Breath samples have recently been found to contain as many as 3000 exhaled volatile organic compounds. These organic compounds are generated through a variety of cellular biochemical processes and are measurable by a number of technologies, well described in this article. On the basis of this premise, exhaled breath fingerprints (volatile organic compound signatures) have proved useful in the differentiation of benign from malignant nodules, which remains a substantial unmet need. As our audience is well aware, lung cancer is the leading cause of cancer-related death in the world, only about 15\% of all lung cancer cases are diagnosed as early stage, and the early detection of lung cancer was shown to decrease lung cancerspecific mortality by $20 \%$ in the landmark National Lung Screening Trial. Among the abnormal results obtained by low-dose computed tomography screening in this trial, $96.4 \%$ were false-positive results, and many of these led to invasive diagnostic procedures. One obvious benefit of a breath print analysis that could discriminate benign and malignant pulmonary nodules is increased accuracy of lung cancer screening and reduced number of unnecessary diagnostic procedures. This is only one of many applications of breath print analyses, however, and this technology has a number of other potential applications in pulmonology, thoracic oncology, and other disciplines, as described in this proceeding. The reader is promised an enjoyable ride through e-nose technology.

\section{Bryan M. Burt, $M D$}

Researchers and clinicians dealing with lung cancer inevitably focus their attention on innovative treatments that can change the fate of our patients. Early diagnosis remains a myth, because lung cancer screening is still plagued by false-positive results and the assessment of the tumor type

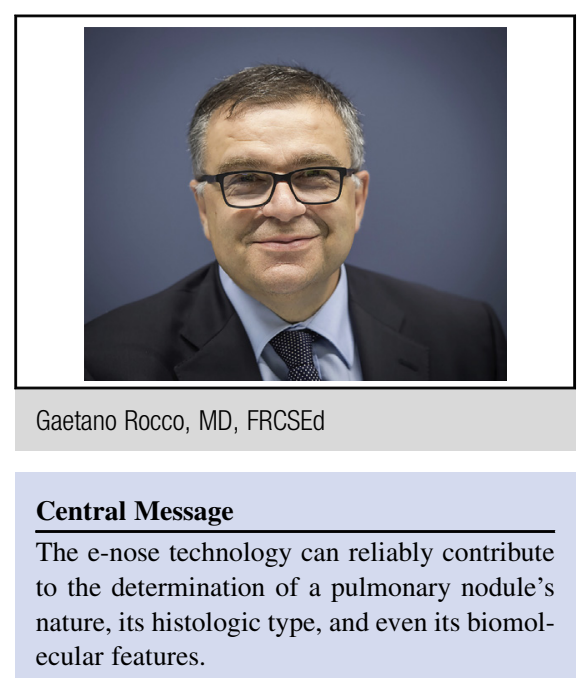

See Editorial Commentary page 2626.

requires some form of invasive modality of tumor biopsy, which at times cannot be tolerated because of the patients' often compromised condition. ${ }^{1-3}$ Liquid biopsy on patients with localized tumors can detect circulating DNA in as many as $55 \%$ of plasma samples, but this percentage is bound to increase with tumor stage. ${ }^{4,5}$ As a consequence, promising therapeutic modalities (eg, stereotactic body radiation) are often administered to patients without histologic confirmation, solely on the basis of clinical algorithms predictive of malignancy. ${ }^{6}$ Video-assisted thoracoscopic surgery itself does not rely consistently on preoperative cytohistologic diagnosis, and minimally invasive lung resections can provide both diagnosis and cure at the same time. ${ }^{7}$ Nevertheless, the reported rate of videoassisted thoracoscopic surgery performed for nodules confirmed as benign at final pathology can be as high as $10 \%$ to $11 \%$. $^{7,8}$ The wide spectrum of disease stages of lung cancer may suggest different pathways to obtain diagnosis of histotype or to detect tumor or immune system markers for individualized treatment. ${ }^{9}$

Volatile organic compounds (VOCs) are chemical structures generated by cellular metabolism and exchanged from tissue to blood and subsequently with the inhaled air in the alveoli. $^{10,11}$ Strictly speaking, VOCs are markers (signatures) of cellular activity present in the exhaled breath. ${ }^{12}$ These compounds can be studied from a quantitative standpoint by using gas chromatography-mass spectrometry, which can provide the exact concentration of 
each compound relative to a standard population. ${ }^{13,14}$ Albeit promising, this quantitative breath analysis has not been able to yield a set of lung cancer-specific VOCs, with the possible exception of 4 recently described carbonyl compounds. ${ }^{15}$ Currently, the electronic nose (e-nose) assessment technology includes 4 modalities, each with distinct advantages and drawbacks ${ }^{16,17}$ (Figure 1). These modalities, which can be used for qualitative analysis, are infrared spectrometry, gas chromatography-mass spectrometry, solid-state sensors, and mass spectrometry. ${ }^{16,17}$ An example of qualitative analysis of exhalates is the use of gas chromatography-mass spectrometry in a fingerprinting mode. ${ }^{13}$ More recently, a multisensorial platform (BIONOTE) has been proposed, which includes an innovative type of e-nose technology ${ }^{18}$ (Figure 2). In this procedure, the exhalate is collected early in the morning from the patient, who is invited to breathe through a device (Pneumopipe, EU patent EP2641537 [A1]2013-09-25) that traps the VOCs onto an absorbent cartridge. ${ }^{18}$ The cartridge then undergoes thermal desorption (ie, dissolution at high temperatures) to recapture the VOCs, which are then exposed to gas sensor arrays. ${ }^{18}$ In this e-nose modality, gas sensor arrays are composed of quartz crystals microbalance (QCM) with anthocyanin-coated gold electrodes characterized by a baseline oscillation frequency. ${ }^{19}$ Once exposed to the gas sensor arrays, the VOCs induce a mass change on sensors, which translates into a change of their baseline oscillation frequency (ie, sensor activation). Through sensor activation, a pattern of sensor signals - fingerprints ${ }^{13,18}$ - is generated, in a similar fashion to the "combinatorial selectivity" that enables natural olfaction to distinguish multiple different odors. ${ }^{13,18}$ Data analysis and classification between groups of VOCs patterns are performed with a mathematical model that is based on a multivariate test such as partial least square discriminant analysis. $^{13,18,20}$ In 2016, a group of Italian investigators (including me) reported on 100 individuals subjected to lung cancer screening in whom a suspicious lung nodule was identified. $^{18}$ These individuals underwent e-nose testing in an effort to differentiate between healthy individuals and those with lung cancer. ${ }^{18}$ The results were encouraging, with sensitivity, specificity, positive predictive value, and negative predictive value of $86 \%, 95 \%, 83 \%$, and $96 \%$, respectively. ${ }^{18}$ Reportedly, irrespective of the sampling technique used in the e-nose technology, exhalate collection and subsequent processing may take as long as 20 minutes, with a reported cost per patient of about $€ 10$. ${ }^{18}$

The paper by Shlomi and coworkers ${ }^{21}$ on the use of nanoarray sensors for breath analysis published in the October 2017 issue of the Journal of Thoracic Oncology has the

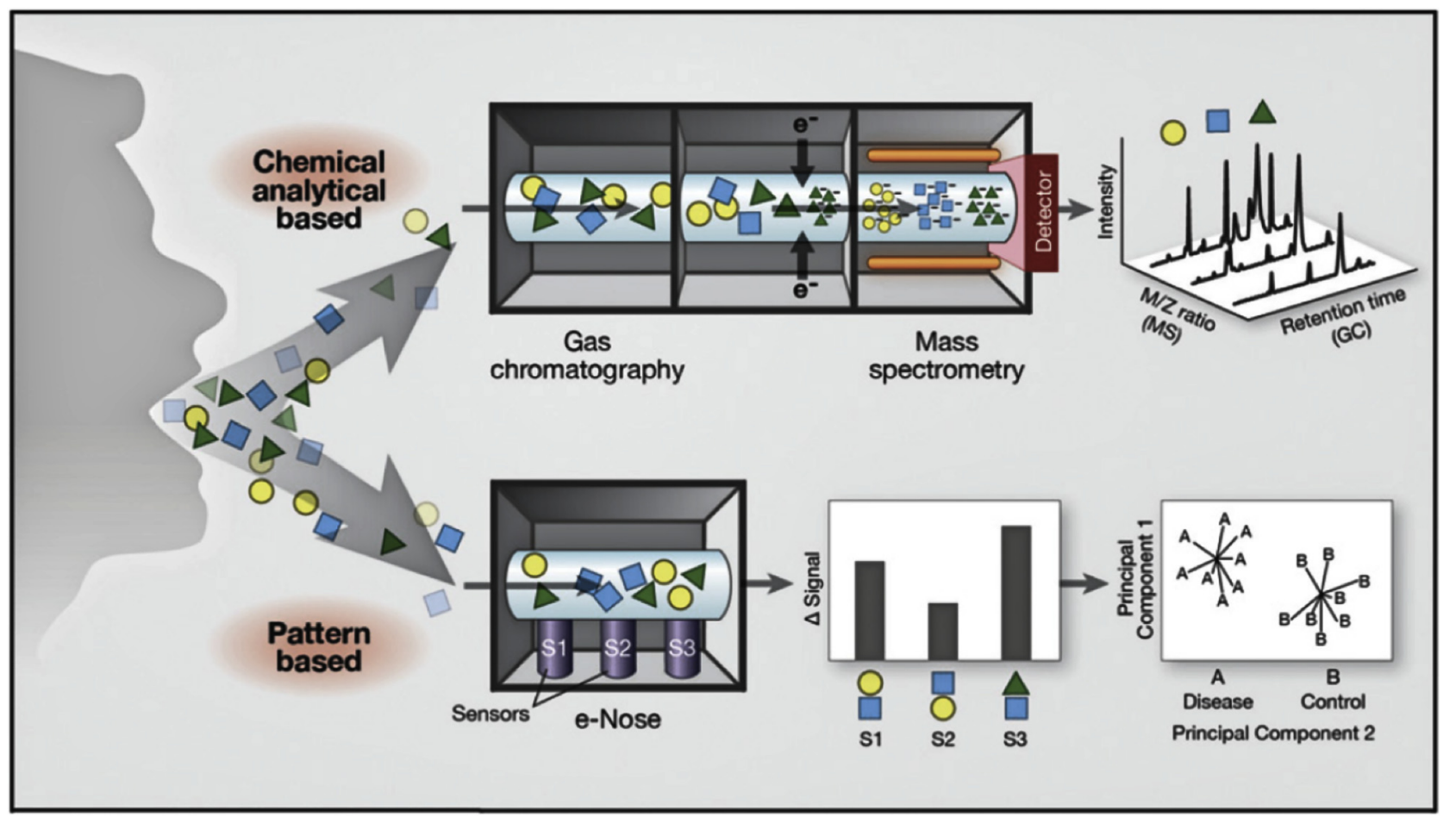

FIGURE 1. The two main methods of assessment of the volatile organic compounds. The analytic methods aim at identifying the single components by comparing them with known compounds in a reference library, whereas the e-nose technology aims at delineating volatile organic compound pattern recognition algorithms to classify each individual patient profile. $M / Z$, Mass-to-charge ratio; $M S$, mass spectrography; $G C$, gas chromatography. (Reproduced with permission from: van der Schee MP, Paff T, Brinkman P, van Aalderen WMC, Haarman EG, Sterk PJ, et al. Breathomics in lung disease. Chest. 2015; 147:224-31.) 


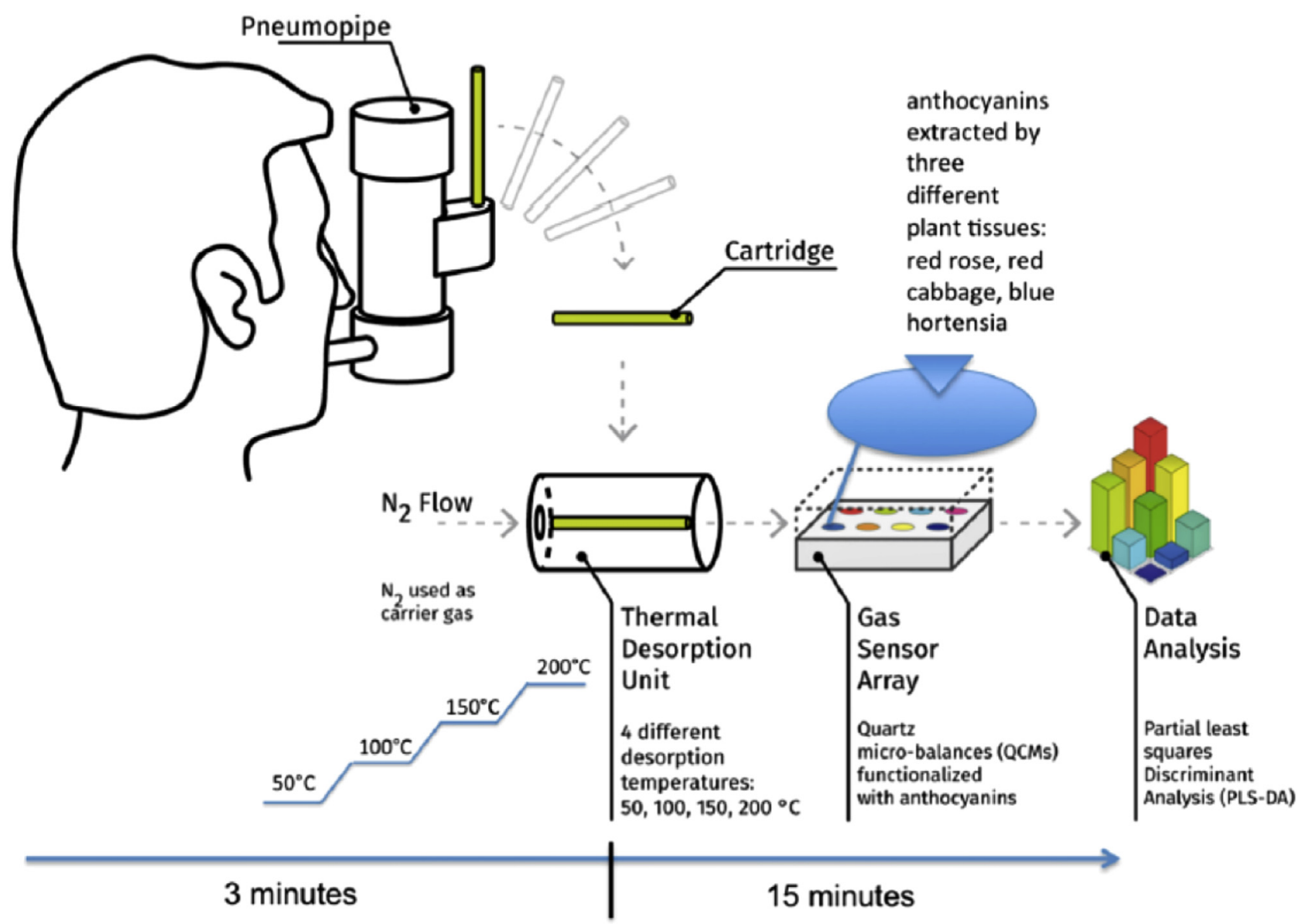

FIGURE 2. The BIONOTE sensorial platform. After collection of exhaled breath into the Pneumopipe and transfer through a Tenax cartridge (Buchem BV, Apeldoorn, The Netherlands) into the thermal desorption unit, volatile organic compounds are exposed to gas sensor microarrays. The mass alteration induced by the volatile organic compounds will induce a modification of the baseline oscillation frequency in the quartz microbalances $(Q C M)$, thus generating the breath print, which is then analyzed with partial least squares discriminant analysis (PLS-DA), as described in the text. (Modified from: Pennazza G, Santonico M, Scarlata S, Santangelo S, Grasso S, Zompanti A, et al. A non invasive sensor system for the screening of non obstructive sleep apnea syndrome. Proceedings. 2017;1:426. Reproduced with permission and under the terms and conditions of Creative Commons Attribution [CC BY] license [http:// creativecommons.org/licenses/by/4.0/].)

distinct merit of furthering the research in this field. Indeed, this study of 119 patients focused on the possibility for the e-nose technology not only to distinguish between malignant and benign nodules but also to determine its potential endothelial growth factor receptor positivity. ${ }^{21}$ The separation between malignant and benign nodules was done with overall accuracy, positive predictive value, and negative predictive value of $87 \%, 88 \%$, and $87 \%$, respectively. ${ }^{21}$ In addition, an accuracy of $83 \%$, a sensitivity of $79 \%$, and a specificity of $85 \%$ were found when endothelial growth factor receptor positivity was assessed on the basis of specific nanoarray sensor features. ${ }^{21}$

Apart from the use of nanotechnology in manufacturing the gas sensors, the main difference in the e-nose technologies presented by Shlomi and coworkers ${ }^{21}$ compared with the one used by the Italian group ${ }^{18}$ resides in the exhalate collection modality (GaSampler polyethylene bags
[QuinTron Instrument Company, Inc, Milwaukee, Wis] vs Pneumopipe), which may not represent a trivial difference because the potential implications for gas preservation and contamination. ${ }^{18,21}$ Nevertheless, the work by Shlomi and coworkers ${ }^{21}$ demonstrates that the use of the e-nose represents today another potentially fruitful application of nanotechnology to thoracic surgery. ${ }^{21,22}$

The prospective advantages of the introduction into clinical practice of the e-nose technology seem obvious: (1) as a diagnostic tool to verify smoking cessation indirectly in patients enrolled in lung cancer screening programs, since the e-nose can assess fingerprints of chronic obstructive pulmonary disease ${ }^{23}$; (2) as a diagnostic tool serving the purpose of identifying high-risk individuals to be subjected to lowdose computed tomographic scanning in the setting of a lung cancer screening program ${ }^{15,18}$; (3) as a confirming test before scheduling an invasive procedure for a patient 
with suspected pulmonary nodule ${ }^{18,21}$; (4) in the postsurgical follow-up protocols to decide whether and when to proceed to computed tomographic scan or positron emission tomography ${ }^{14,18,24}$; (5) as a noninvasive method to support the diagnosis of malignancy indicated by clinical algorithms, as is often the case when biopsy is not feasible and the patient needs to be subjected to alternative treatments to surgery, such as stereotactic body radiation ${ }^{18,21,25}$; and (6) as a noninvasive method to identify lung cancer-related genetic mutations. ${ }^{21,26}$

There are still, however, limitations to the widespread use of the e-nose that impose caution in the interpretation of the currently available evidence from the literature. There are major hurdles opposing a more diffuse clinical implementation of this technology. The relatively small numbers of the populations subjected to e-nose evaluation and the lack of a standardized and miniaturized device enabling sample collection and data analysis in real time represent the most obvious flaws. In addition, alterations in the composition of the exhaled breath may affect VOC analysis. Examples of such alterations could result from the previous use of drugs, especially chemotherapy agents, and the presence of concurrent viral or bacterial infection. ${ }^{27}$ In this setting, the ability of e-nose technology to separate lung cancer from chronic obstructive pulmonary disease has been already reported. ${ }^{27}$ In the future, the possibility of applying the same principles of the e-nose to the assessment of fingerprints in biologic fluids through the so-called e-tongue is being explored. ${ }^{19}$ The e-tongue can be used to confirm e-nose and liquid biopsy findings, thus enhancing the overall diagnostic ability in the "no touch" diagnostic lung cancer setting.

In conclusion, the e-nose technology represents a promising, noninvasive modality of obtaining histologic diagnosis of a pulmonary nodule and even of assessing its biomolecular profile. The possible clinical applications of this technology are manifold, but they need to be verified against its current significant limitations.

\section{Conflict of Interest Statement}

Authors have nothing to disclose with regard to commercial support.

\section{References}

1. Field JK. Perspective: the screening imperative. Nature. 2014:513:S7.

2. Chudgar NP, Bucciarelli PR, Jeffries EM, Rizk NP, Park BJ, Adusumilli PS, et al. Results of the national lung screening trial: where are we now? Thorac Surg Clin. 2015;25:145-53.

3. Heuvelmans MA, Oudkerk M, de Bock GH, de Koning HJ, Xie X, van Ooijen PM, et al. Optimisation of volume-doubling time cutoff for fastgrowing lung nodules in CT lung cancer screening reduces false-positive referrals. Eur Radiol. 2013;23:1836-45.

4. Karachaliou N, Mayo-de-Las-Casas C, Molina-Vila MA, Rosell R. Real-time liquid biopsies become a reality in cancer treatment. Ann Transl Med. 2015;3:36.
5. Fenizia F, De Luca A, Pasquale R, Sacco A, Forgione L, Lambiase M, et al. EGFR mutations in lung cancer: from tissue testing to liquid biopsy. Future On col. 2015;11:1611-23.

6. Louie AV, Senan S, Patel P, Ferket BS, Lagerwaard FJ, Rodrigues GB, et al. When is a biopsy-proven diagnosis necessary before stereotactic ablative radiotherapy for lung cancer?: a decision analysis. Chest. 2014; 146:1021-8.

7. Petersen RH, Hansen HJ, Dirksen A, Pedersen JH. Lung cancer screening and video-assisted thoracic surgery. J Thorac Oncol. 2012;7:1026-31.

8. Kuo E, Bharat A, Bontumasi N, Sanchez C, Zoole JB, Patterson GA, et al. Impact of video-assisted thoracoscopic surgery on benign resections for solitary pulmonary nodules. Ann Thorac Surg. 2012;93:266-72; discussion 272-3.

9. Rocco G, Morabito A, Leone A, Muto P, Fiore F, Budillon A. Management of non-small cell lung cancer in the era of personalized medicine. Int J Biochem Cell Biol. 2016;78:173-9.

10. Phillips M, Gleeson K, Hughes JM, Greenberg J, Cataneo RN, Baker L, et al. Volatile organic compounds in breath as markers of lung cancer: a cross-sectional study. Lancet. 1999;353:1930-3.

11. Haick H, Broza YY, Mochalski P, Ruzsanyi V, Amann A. Assessment, origin, and implementation of breath volatile cancer markers. Chem Soc Rev. 2014;43: 1423-49.

12. Capuano R, Santonico M, Pennazza G, Ghezzi S, Martinelli E, Roscioni C, et al The lung cancer breath signature: a comparative analysis of exhaled breath and air sampled from inside the lungs. Sci Rep. 2015;5:16491.

13. D’Amico A, Di Natale C, Falconi C, Martinelli E, Paolesse R, Pennazza G, et al Detection and identification of cancers by the electronic nose. Expert Opin Med Diagn. 2012;6:175-85

14. Schumer EM, Black MC, Bousamra M II, Trivedi JR, Li M, Fu XA, et al Normalization of exhaled carbonyl compounds after lung cancer resection. Ann Thorac Surg. 2016;102:1095-100.

15. Schumer EM, Trivedi JR, van Berkel V, Black MC, Li M, Fu XA, et al High sensitivity for lung cancer detection using analysis of exhaled carbonyl compounds. J Thorac Cardiovasc Surg. 2015;150:1517-22; discussion 1522-4.

16. InfoMetrix. Application Notes: Electronic Nose Instrumentation (1999) Available at: https://chemometrix.files.wordpress.com/2014/07/20-0199_ enosecompan.pdf. Accessed September 17, 2017.

17. Nardi-Agmon I, Peled N. Exhaled breath analysis for the early detection of lung cancer: recent developments and future prospects. Lung Cancer (Auckl). 2017;8 $31-8$.

18. Rocco R, Incalzi RA, Pennazza G, Santonico M, Pedone C, Bartoli IR, et al. BIONOTE e-nose technology may reduce false positives in lung cancer screening programmes. Eur J Cardiothorac Surg. 2016;49:1112-7; discussion 1117

19. Santonico M, Pennazza G, Grasso S, D'Amico A, Bizzarri M. Design and test of a biosensor-based multisensorial system: a proof of concept study. Sensors (Basel). 2013;13:16625-40.

20. Pennazza G, Santonico M, Finazzi Agrò A. Narrowing the gap between breath printing and disease diagnosis, a sensor perspective. Sens Actuators B Chem 2012;179:270-5

21. Shlomi D, Abud M, Liran O, Bar J, Gai-Mor N, Ilouze M, et al. Detection of lung cancer and EGFR mutation by electronic nose system. J Thorac Oncol. 2017;12 1544-51.

22. Hofferberth SC, Grinstaff MW, Colson YL. Nanotechnology applications in thoracic surgery. Eur J Cardiothorac Surg. 2016;50:6-16.

23. Incalzi RA, Pennazza G, Scarlata S, Santonico M, Petriaggi M, Chiurco D, et al. Reproducibility and respiratory function correlates of exhaled breath fingerprint in chronic obstructive pulmonary disease. PLoS One. 2012;7: e45396.

24. Broza YY, Kremer R, Tisch U, Gevorkyan A, Shiban A, Best LA, et al. A nanomaterial-based breath test for short-term follow-up after lung tumor resection. Nanomedicine. 2013;9:15-21.

25. Barash O, Peled N, Tisch U, Bunn PA Jr, Hirsch FR, Haick H. Classification of lung cancer histology by gold nanoparticle sensors. Nanomedicine. 2012;8: 580-9.

26. Peled N, Barash O, Tisch U, Ionescu R, Broza YY, Ilouze M, et al. Volatile fingerprints of cancer specific genetic mutations. Nanomedicine. 2013;9:758-66.

27. D’Amico A, Pennazza G, Santonico M, Martinelli E, Roscioni C, Galluccio G et al. An investigation on electronic nose diagnosis of lung cancer. Lung Cancer 2010;68:170-6. 